\title{
Variation and significance of secretory immunoglobulin A, interleukin 6 and dendritic cells in oral cancer
}

\author{
SUXIN ZHANG, XIN ZHANG, KE YIN, TIANKE LI, YANG BAO and ZHONG CHEN \\ Department of Stomatology, The Fourth Hospital of Hebei Medical University, Shijiazhuang, Hebei 050011, P.R. China
}

Received March 7, 2016; Accepted December 2, 2016

DOI: $10.3892 / \mathrm{ol} .2017 .5703$

\begin{abstract}
The present study aimed to determine changes in the concentration of secretory immunoglobulin A (SIgA) and interleukin 6 (IL-6) in the saliva of patients with oral cancer, to evaluate the abnormal expression of cluster of differentiation (CD) 1a, CD83, CD80 and CD86 on dendritic cells (DCs) of oral cancer tissues and to discuss the interaction between SIgA, IL-6 and DCs in oral cancer. A total of 40 patients between 27 and 70 years of age, median age 52 years, with primary oral cancer were enrolled in the present study, and a group of 20 healthy male and female volunteers was used as the control group. The concentration of SIgA and IL-6 in the saliva of the preoperative patients was determined by ELISA. The expression levels of CD1a, CD83, CD80 and CD86 were detected by immunohistochemistry and flow cytometry, which was performed on histopathological sections from paraffin-embedded tumor and corresponding adjacent control tissues. The specimens were assessed using the semi-quantitative immunoreactive score (IRS). The concentration of SIgA in the saliva from patients with oral cancer decreased, whereas the IL-6 level significantly increased compared with the control subjects $(\mathrm{P}<0.05)$. In addition, the decrease of SIgA level and increase of IL-6 level exhibited a negative correlation $(\mathrm{r}=-0.543, \mathrm{P}<0.05)$. According to the IRS score, the expression levels of CD1a, CD83, CD80 and CD86 in the cancer tissue were lower than the expression levels of the control group $(\mathrm{P}<0.05)$. Furthermore, the expression of CD80 and CD86 exhibited no correlation with histological grade or pathological type $(\mathrm{P}>0.05)$, but exhibited a negative correlation with clinical stage and lymph node metastasis $(\mathrm{P}<0.05)$. The concentration of SIgA and IL-6 in saliva may be used as an auxiliary diagnostic indicator for oral cancer. The detection of CD80 and CD86 expressed on DCs in oral cancer tissue may be useful for the diagnosis and evaluation of the prognosis of tumors. The present study hypothesized that the use of SIgA
\end{abstract}

Correspondence to: Dr Zhong Chen, Department of Stomatology, The Fourth Hospital of Hebei Medical University, 361 Zhongshan E Road, Shijiazhuang, Hebei 050011, P.R. China

E-mail: 649589734@qq.com

Key words: oral cancer, salivary secretory immunoglobulin A, interleukin 6 , dendritic cell, tumor immunity vaccines or IL- 6 inhibitors may be useful for reversing the immune deficiency associated with DCs in oral cancer.

\section{Introduction}

Oral cancer is the sixth most common type of human cancer in the world, with an incidence of $\sim 300,300$ per year (1). Although significant progress has been made on multidisciplinary collaboration and systemic treatment, the long-term survival rates and quality of life of patients remain poor (2). The main cause for the poor prognosis of oral cancer is the high local recurrence rate associated with this type of cancer (3). The development of effective methods to prevent the repeated invasion of cancer cells, inhibiting the proliferative phase is therefore required. Immune status has been revealed to serve an important role in interfering with tumor progression, and dendritic cells (DCs), the most efficient type of antigen presenting cells, have become the focus of investigation into immune regulation (4). The regulatory cytokines of DCs, such as interleukin (IL)-6, IL-2 and IL-10, serve important roles in maintaining mucosal homeostasis (5).

Human saliva may be used for the diagnosis of various pathological states. Secretory immunoglobulin A (SIgA), as a prominent antibody in saliva, possesses an important reference value in local mucosal immunity. The SIgA molecule, a heterodimer, is composed of $2 \operatorname{Ig} \mathrm{A}$ monomers, each containing 2 light chains and 2 heavy chains, $1 \mathrm{~J}$ chain and 1 secretory component (SC) (6). SIgA exhibits the characteristics of long stability and high detection rate in saliva, which can reflect the immune state of the oral cavity (7). As the main factor in the process of mucosal response (8), SIgA has been found to exhibit cooperation with DCs in vitro, and IL-6 may be associated with the changes of SIgA and DCs (9).

The present study investigated the levels of SIgA and IL-6 and changes with respect to DC surface markers during tumor mass formation, and explored the association between the aforementioned changes and the clinical and pathological features of oral tumors. The association between SIgA and IL-6 in the saliva of patients with oral cancer was examined, and the influence of SIgA and IL-6 on DCs was suggested.

\section{Materials and methods}

Patients and tissues. The patients with oral cancer were all inpatients for surgical treatment in the Fourth Hospital 
of Hebei Medical University, Hebei, China, between June 2010 and December 2011. The characteristics of the patients are shown in Table I. Unstimulated saliva $(3 \mathrm{ml})$ from each patient was collected prior to therapy. The saliva was centrifuged at $1,500 \times \mathrm{g}$ for $30 \mathrm{~min}$ at $4^{\circ} \mathrm{C}$, and the supernatant was preserved in a refrigerator at $-80^{\circ} \mathrm{C}$. The cancer and corresponding adjacent control tissues were obtained from the surgery. The tissues were divided into two parallel sections. The first section was immediately ground into single-celled levitation liquid according to previous protocol (10) until flow cytometry detection. The other section was fixed in formalin and embedded into paraffin. Histological typing of the tumor tissues was carried out on the basis of resected specimens in the Department of Pathology, Hebei Medical University, Shijiazhuang, China. The present study was approved by the Ethics Committee of Hebei Cancer Institute, Hebei, China, and informed consent was obtained from all the recruited patients.

SIgA and IL-6 immunoassay. The concentration of SIgA and IL-6 in saliva was measured with the SIgA and IL-6 Quantikine ELISA (DB-100; R\&D Systems, Inc., Minneapolis, MN, USA) following the protocol of the manufacturer. The samples were incubated in duplicate on microtiter plates coated with anti-SIgA monoclonal antibody (cat. no. 11137A12) or anti-IL-6 (cat. no. 11134A09) antibodies (both R\&D Systems, Inc.) for $2 \mathrm{~h}$ at room temperature. The plates were then washed with the washing buffer of the ELISA kit to remove the unbound antibodies. Subsequent to the incubation at $25^{\circ} \mathrm{C}$ for $60 \mathrm{~min}$ of the conjugate solution, horseradish peroxidase-conjugated reagent, and the biotin solution of the ELISA kit was added. Color development was stopped subsequent to 20-25 min, depending on the assay (11). The ELISA assay results were read using a VersaMax microplate spectrophotometer (Molecular Devices, LLC, Sunnyvale, CA, USA) set at $450 \mathrm{~nm}$.

Immunohistochemical staining (IHC). The expression levels of cluster of differentiation (CD)1a, CD83, CD80, and CD86 were determined by the immunostaining method, which was performed on parallel histopathological sections from paraffin-embedded tumor sections and corresponding adjacent control tissues. The tissue sections were deparaffinized in xylene, rehydrated in graded alcohol and washed in water. Endogenous peroxidase activity was blocked with $\mathrm{H}_{2} \mathrm{O}_{2}$ treatment. Antigen retrieval was achieved by incubation in $10 \mathrm{mM}$ boiling sodium citrate buffer for $15 \mathrm{~min}$ and non-specific binding was blocked by treating the sections with $1.5 \%$ horse serum (cat. no. 13011-8611; Hangzhou Biotechnology Co., Ltd., Hangzhou, China) for $10 \mathrm{~min}$. Monoclonal antibodies specific for CD1a (cat. no. BA-2731; dilution, 1:100), CD83 (cat. no. BS-2519R; dilution, 1:100), CD80 (cat. no. BS-1479R; dilution, 1:100), and CD86 (cat. no. BS-1035R; dilution, 1:100) were obtained from Beckman Coulter, Inc., Brea, CA, USA. The slides were incubated with primary antibodies at $4^{\circ} \mathrm{C}$ overnight. The VECTASTAIN ABC kit was used for the final staining (cat. no. 948868A; Vector Laboratories, Inc., Beijing, China). The compound 3,3'-diaminobenzidine (Sigma-Aldrich; Merck Millipore, Billerica, MA, USA) was used as the chromogen. The slides were counterstained with hematoxylin. For the negative control, the primary antibody was replaced with mouse IgG (Beckman Coulter, Inc.), and slides with control
Table I. Characteristics of the patients.

Clinical feature

No. of patients

\begin{tabular}{lc}
\hline Gender & \\
Male & 18 \\
Female & 22 \\
Age (years) & \\
Maximum & 70 \\
Minimum & 27 \\
Median & 52 \\
Clinical stages & \\
Phases I, II & 26 \\
Phase III, IV & 14 \\
Pathological type & \\
Squamous cell carcinoma & 30 \\
Adenocarcinoma & 10 \\
Lymphatic metastasis & \\
Yes & 11 \\
No & 29 \\
\hline
\end{tabular}

oral mucosa were used as the positive control. Each immunoreactive endothelial cell cluster in contact with the selected field was counted as an individual vessel. The specimens were assessed using the semi-quantitative immunoreactive score (IRS). The IRS was calculated by multiplying the staining intensity ( 0 , no staining; 1 , weak staining; 2 , moderate staining; and 3 , strong staining) by the percentage of positively stained cells $(0,<10 \%$ cells stained; $1,11-50 \%$ cells stained; $2,51-80 \%$ of cells stained; and $3,>81 \%$ cells stained). The criterion for positive staining was an IRS score of $\geq 2$ (12).

Flow cytometry. Formalin-fixed and fresh tissues were cut into sections, rubbed gently with plastic forceps and rinsed with physiological saline. The suspension was filtered with a 300-mesh copper grid to remove the block, and the cell suspension was centrifuged at $150 \times g$ for $2 \mathrm{~min}$. The cells $\left(1 \times 10^{6} / \mathrm{ml}\right)$ were collected and washed twice in PBS. A flow cytometer (Epics-XL II; Beckman Coulter, Inc.) was used to determine the cell surface expression of CD1a, CD83, CD86 and CD80 according to previous protocol $(13,14)$. Briefly, mononuclear cells were stained using monoclonal antibodies to CD1a, CD83, CD86, CD80 (Beckman Coulter, Inc., Brea, CA, USA) and appropriate $\operatorname{IgG}$ isotype controls. Cells were kept at $4^{\circ} \mathrm{C}$ for $30 \mathrm{~min}$, and filtered using a 500-mesh copper grid. The samples were analyzed using a FACSCalibur cytometer and CellQuest Pro software (version 5.1; BD Biosciences, Franklin Lakes, NJ, USA).

Statistical analysis. Statistical analysis was performed using SPSS 13.0 software (SPSS, Inc., Chicago, IL, USA). $\chi^{2}$, Fisher's exact and unpaired $t$-tests were performed according to the data to assess the statistical significance of differences and compare categorical associations. A two-sided $t$-test was used to determine the significance, with $\mathrm{P}<0.05$ considered to indicate a statistically significant difference. 
Table II. Expression of CD1a, CD83, CD80 and CD86 in oral cancer and control tissues.

\begin{tabular}{|c|c|c|c|c|c|c|c|c|}
\hline \multirow{2}{*}{$\begin{array}{l}\text { Surface } \\
\text { molecule }\end{array}$} & \multicolumn{3}{|c|}{ Oral tissue } & \multicolumn{3}{|c|}{ Control tissue } & \multirow[b]{2}{*}{$\chi^{2}$} & \multirow[b]{2}{*}{ P-value } \\
\hline & $-n$ & $+n$ & Positive rate $\%$ & $-n$ & $+n$ & Positive rate $\%$ & & \\
\hline CD1a & 17 & 23 & 57.5 & 2 & 18 & 90.0 & 6.508 & 0.011 \\
\hline CD83 & 19 & 21 & 52.5 & 4 & 16 & 80.0 & 4.266 & 0.039 \\
\hline CD80 & 18 & 22 & 55.0 & 2 & 18 & 90.0 & 7.35 & 0.007 \\
\hline CD86 & 17 & 23 & 57.5 & 3 & 17 & 85.0 & 4.538 & 0.033 \\
\hline
\end{tabular}

$\mathrm{CD}$, cluster of differentiation.

\section{Results}

Levels of SIgA and IL-6 in the saliva. The concentration of SIgA in the saliva of patients with oral cancer was significantly lower than the concentration of the control group $(83.40 \pm 8.25$ vs. $97.47 \pm 5.10 \mu \mathrm{g} / 1 ; \mathrm{t}=-6.875, \mathrm{P}<0.01)$. By contrast, the concentration of IL-6 in the saliva of patients with oral cancer was significantly higher than the concentration of the control group ( $4.86 \pm 1.02$ vs. $1.33 \pm 0.05 \mu \mathrm{g} / \mathrm{l} ; \mathrm{t}=15.422, \mathrm{P}<0.01)$. Furthermore, the concentration of SIgA in the saliva of patients exhibited a negative correlation with the concentration of IL-6 $(r=-0.543$, $\mathrm{P}<0.01)$. The present study found no correlation between the concentrations of SIgA and IL- 6 and patient-associated parameters such as clinical stage, pathological type, histological grade and lymph node metastasis prior to treatment (Fig. 1).

Expression of CDIa, CD83, CD80 and CD86 on DCs. The DCs exhibited irregular shapes and contained dendritic processes, and staining of the surface molecules CD1a, CD83, CD80 and CD86 was detected in the cytoplasm and nuclei. The DCs were mainly located in the basal and spine layers and were distributed uniformly in the control oral mucosa epithelium, whereas the DCs scattered in the cancer tissue and numerous dendritic processes were communicating with the cancer cells (Fig. 2). The frequency of positive expression of CD1a and CD83 in the tumor tissue was $57.5 \%$ (23/40) and $52.5 \%(21 / 40)$, respectively, whereas $90.0 \%(18 / 20)$ and $80.0 \%(16 / 20)$ corresponding control tissue showed a positive expression of CD1a and CD83 (both $\mathrm{P}<0.05$ ). The frequency of positive expression of CD80 and CD86 in the tumor tissue was $55.0 \%(22 / 40)$ and $57.5 \%(23 / 40)$, respectively, whereas $90.0 \%(18 / 20)$ and $85.0 \%$ (17/20) corresponding control tissues showed positive expression of CD80 and CD86 (both $\mathrm{P}<0.05$; Table II).

Expression of DCs markers and their correlation with clinicopathological parameters. As assessed by flow cytometry, the percentages of CD1a and CD83 positive cells in the tumor tissue were $22.28 \pm 7.93$ and $2.09 \pm 1.18 \%$, respectively, a significant decrease compared with the controls $(30.46 \pm 6.79$ and $10.13 \pm 5.99 \%$, respectively; both $\mathrm{P}<0.05$ ). The percentages of CD80 and CD86 positive cells in the tumor tissue were $6.37 \pm 3.23$ and $18.35 \pm 6.02 \%$, respectively, which was also a significant decrease compared with the controls $(15.17 \pm 4.94$ and $31.77 \pm 5.23 \%$, respectively; both $\mathrm{P}<0.05$; Fig. 3).
Fig. 4 shows that there was no significant correlation between tumor characteristics such as the clinical stage, pathological type, histological grade and lymph node metastasis and the expression of CD1a and CD83 in the tumor tissue (both $\mathrm{P}>0.05$ ). However, although the expression of CD80 and CD86 exhibited no association with the pathological type and histological grade of the tumor tissue (both $\mathrm{P}>0.05$ ), the expression of CD80 and CD86 was associated with the clinical stage $(r=-0.625$ and $\mathrm{P}=0.020, \mathrm{r}=-0.610$ and $\mathrm{P}=0.041$, respectively) and lymph node metastasis $(\mathrm{r}=-0.613$ and $\mathrm{P}=0.036, \mathrm{r}=-0.594$ and $\mathrm{P}=0.024$, respectively) of the tumor tissue. Thus, as the correlation analysis illustrated, the expression of CD80 and CD86 was negatively correlated with the lymph node metastasis and clinical stage of the tumor tissue (Fig. 4).

\section{Discussion}

Oral cancer is one of the common malignant types of tumor in the head and neck, which includes tongue, gum, buccal, mouth and palate carcinoma (15). Oral tumors are characterized by a high degree of malignancy, infiltration into adjacent tissues, lymph node metastasis and high recurrence rates (16). Although the current multidisciplinary collaborative therapy is progressing, the long-term survival rate of patients with oral cancer and the quality of life subsequent to treatment remain poor $(17,18)$. The 5 -year survival rate of patients with cervical lymph node metastasis is $<40 \%$ (19). Therefore, early diagnosis and screening of tumor markers requires investigation, with the present study providing preliminary data.

The results of the present study have demonstrated that the concentration of SIgA markedly decreased in the saliva of patients with oral cancer, while the concentration of IL-6 clearly increased, which exhibited no association with the clinical stage, histological grade, pathological type and lymph node metastasis. Shpitzer et al (20) reached a similar conclusion with respect to the reduction of SIgA in patients with cancer, revealing that the level of SIgA decreased by $45 \%$ in mixed saliva from patients with oral squamous cell carcinoma. SIgA is considered to be an exocrine secretion of the IgA antibody and serves a crucial role in mucosal immune system (21). The incidence of malignant tumors in patients with the congenital lack of SIgA has been found to be 20- to 50 -fold higher compared with individuals that do not exhibit the congenital abnormality (22). The mucous membrane of the small intestine exhibits rare malignant tumors, of which 
A
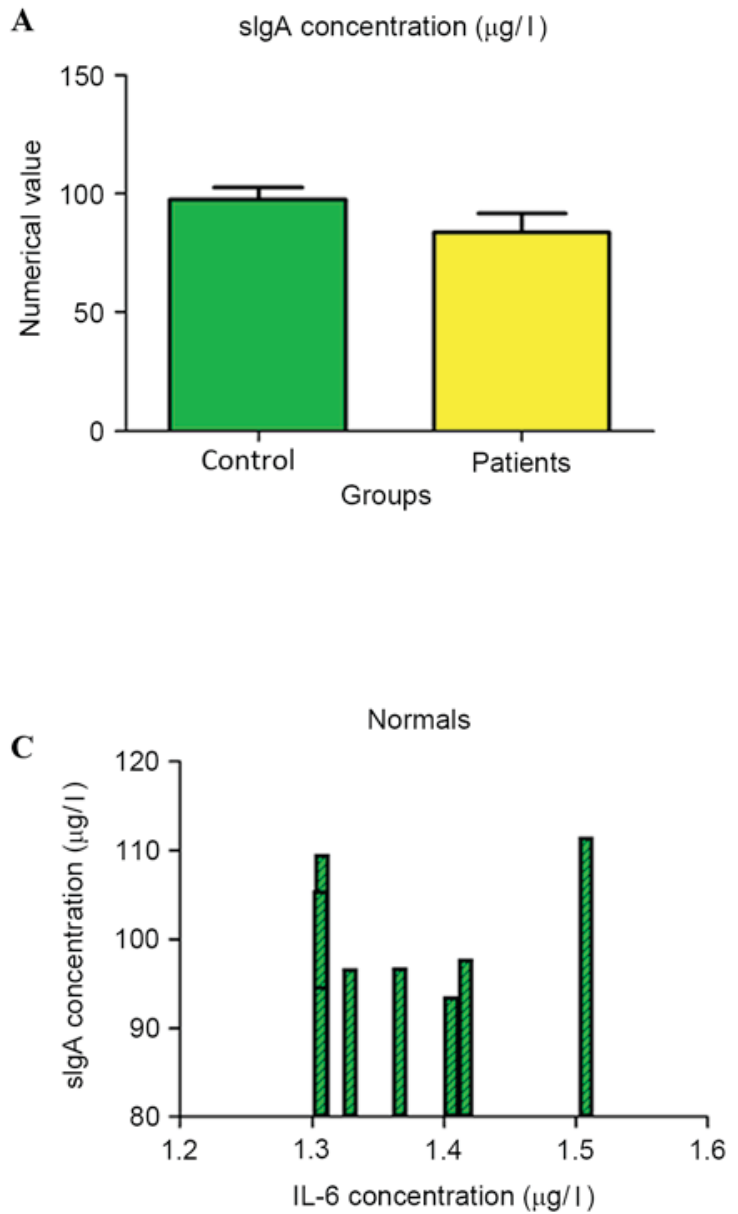

B

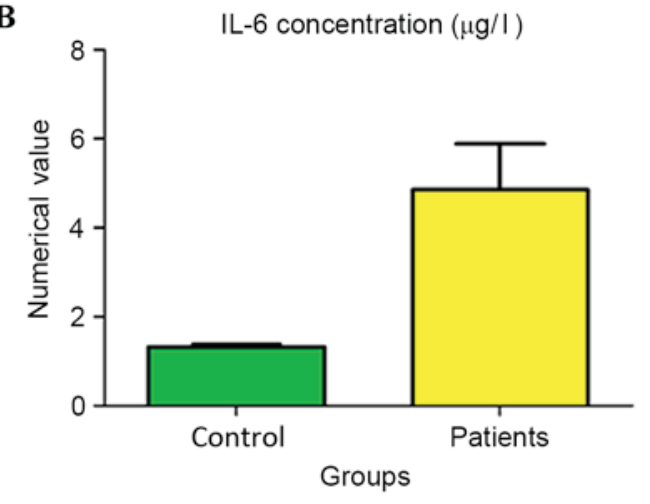

D

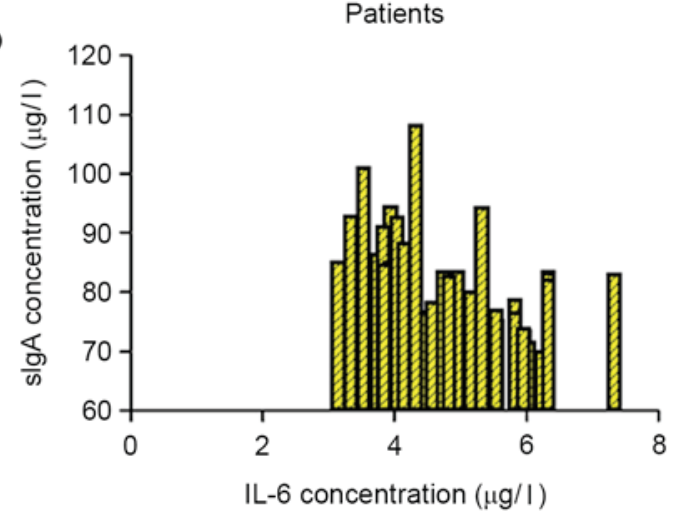

Figure 1. Comparison of SIgA and IL-6 levels in the saliva of control subjects and patients with oral cancer. (A) The concentration of SIgA in the saliva of patients with oral cancer was significantly lower compared with the control group. (B) The concentration of IL-6 in the saliva of patients with oral cancer was significantly higher compared with the control group. The association between SIgA and IL-6 concentration in the saliva of the (C) control group and (D) patients. The concentration of SIgA in the saliva of patients exhibited a negative correlation with the concentration of IL-6. SIgA, secretory immunoglobulin A; IL-6, interleukin 6.

A
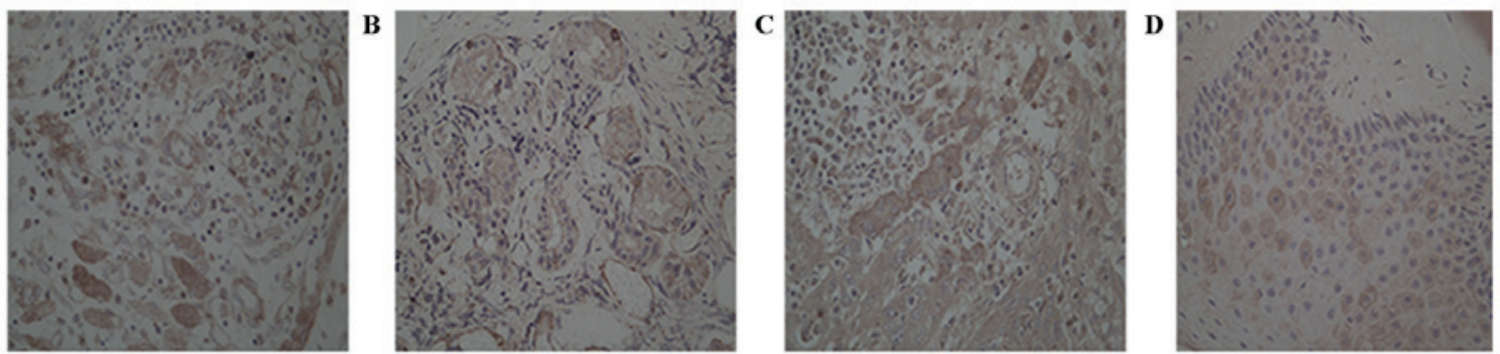

$\mathbf{E}$
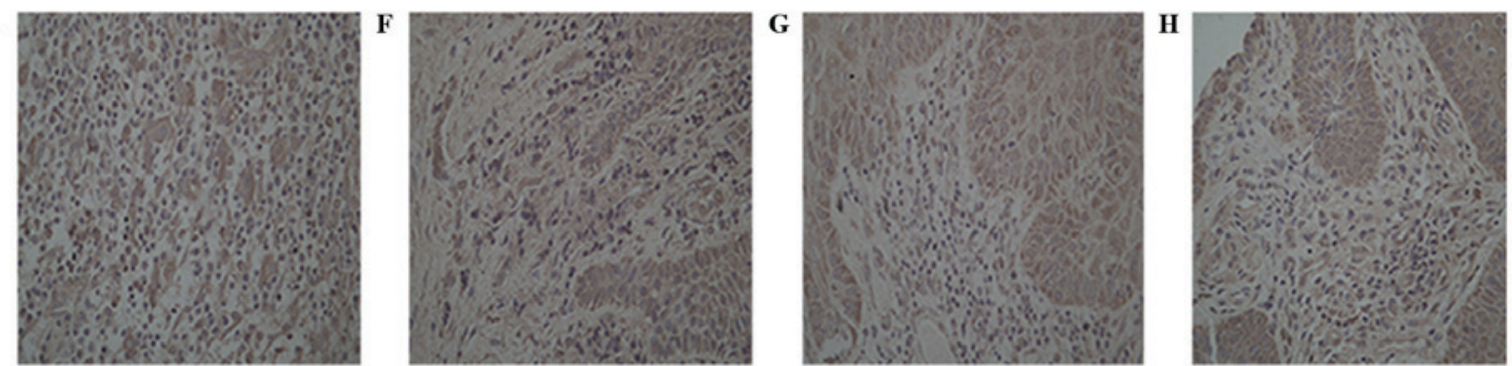

Figure 2. Staining of (A) CD1a in cancer tissue, (B) CD1a in control tissue, (C) CD83 in cancer tissue, (D) CD83 in control tissue, (E) CD80 in cancer tissue, (F) CD80 in control tissue (G) CD86 in cancer tissue and (H) CD86 in control tissue (magnification, x400). The majority of DCs were located in the basal and spinal layers and were distributed uniformly in control oral mucosa epithelium, whereas in the cancer tissue the DCs were scattered irregularly and numerous dendritic processes were communicating with cancer cells. CD80 and CD86 were stained brown in the cytoplasm and nuclei. CD, cluster of differentiation; DCs, dendritic cells. 

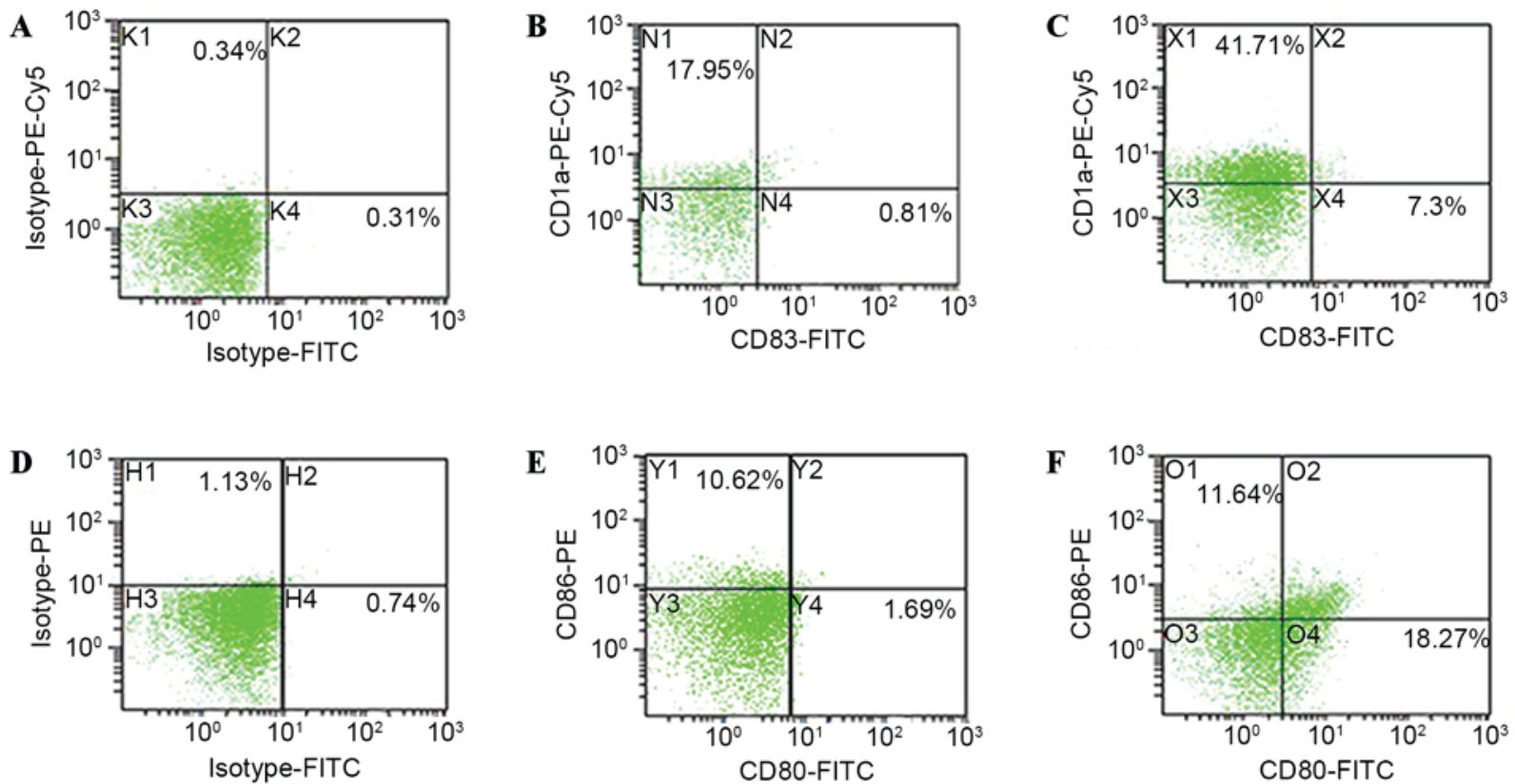

Figure 3. Expression of CD1a, CD83, CD80 and CD86 in control and oral cancer tissues. Flow cytometryy analysis showed that percentage of CD1a, CD83, CD80 and CD86 positive cells was significantly lower in the cancer tissue compared with the control ( $\mathrm{P}<0.05)$. (A) CD1a and CD83 percentages of negative controls, (B) CD1a and CD83 percentages of patients, (C) CD1a and CD83 percentages of healthy individuals, (D) CD80 and CD86 percentages of negative controls, (E) CD80 and CD86 percentages of patients, (F) CD80 and CD86 percentages of healthy individuals. CD, cluster of differentiation; PE, phycoerythricin; FITC, fluorescein isothiocyanate; Cy5, cyanine.

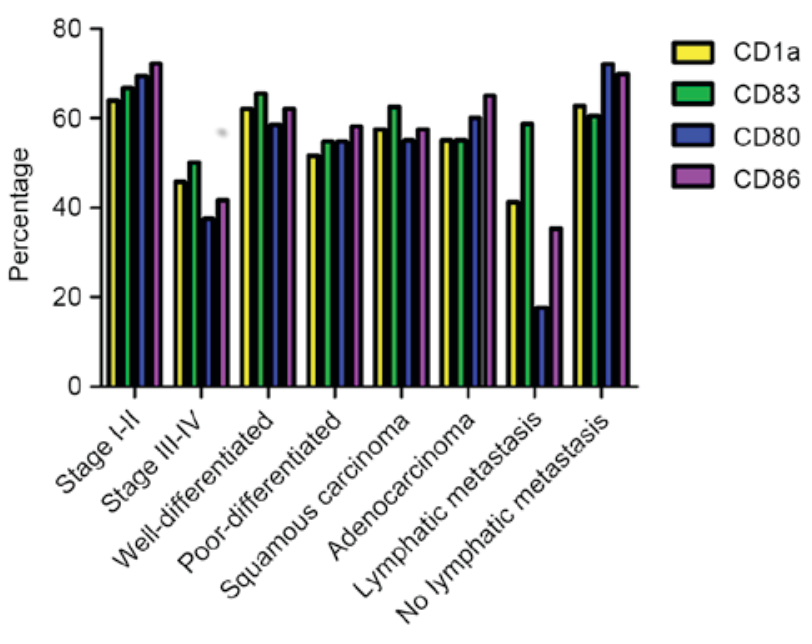

Clinicopathological characteristics

Figure 4. Association between the expression of CD1a, CD83, CD80 and CD86 in oral cancer tissues and different clinical pathological characteristics. No significant correlation was identified between tumor characteristics such as clinical stage, pathological type, histological grade and lymph node metastasis and the expression of CD1a and CD83. The expression of CD80 and CD86 was negatively correlated with lymph node metastasis and clinica stage, but exhibited no correlation with pathological type and histological grade. $\mathrm{CD}$, cluster of differentiation.

one of the important factors is the secretion of $\operatorname{IgA}$ by $>85 \%$ of the cells of the small intestine (23). The reduction in the level of SIgA in saliva subsequent to the occurrence of malignant tumors is not fully understood. The present study proposes that the extracellular hydrolysis of polymeric immunoglobulin receptor (PIgR) may be inhibited when tumors occur, and it is difficult for the SC of SIgA to connect with the corresponding ligand. Therefore, the concentration of SIgA declines. The hypothesis of the present study is based on the findings of Johansen et al (24), who reported that PIgA secretion decreased in PIgR knockout mice, and as a result the level of SIgA in external secretions was significantly reduced. The mechanism for the decrease in the level of SIgA requires additional investigation.

IL-6 is a multifunctional cytokine, which serves different roles in different target cells, affecting the proliferation and differentiation of malignant cells during the occurrence and development of tumors (25-27). The data of the present study showed that the concentration of IL-6 in saliva increased markedly, consistent with the results from a multiplexed immunobead-based assay by Arellano-Garcia et al (28). Sato et al (29) proposed that IL-6 inactivates p53 by inhibiting p53 promoter activity, thereby affecting cell proliferation and apoptosis. Notably, the present study found a negative correlation between the level of SIgA and IL-6 in the saliva of patients with oral cancer, which indicates that during oral cancer occurrence and proliferation, the ability of abnormal cells to synthesize and secrete IL-6 is enhanced, whereas the ability to synthesize and secrete $\mathrm{SIgA}$ is inhibited. Previous studies also stated that IL- 6 serves a key role in the localization of the effector site, terminal differentiation and the proliferation of plasma cells that secrete $\operatorname{IgA}$ in mucosal surfaces $(30,31)$. A reduction in the level of SIgA weakens the ability of the protein to restrain and block antigens (32-34), therefore mucosal immunity is reduced and tumors appear. Additionally, the continuous production of IL- 6 by the tumor cells accelerated abnormal proliferation and differentiation, thus the number of neoplasms increased. Therefore, it is theorized that SIgA and IL-6 may be used as auxiliary diagnostic indicators for oral cancer. 
The second finding of the present study is associated with the immunodeficiency of DCs in oral cancer tissue and the abnormal expression of CD1a, CD83, CD80, and CD86. Among these markers, the expression of CD80 and CD86 exhibited a negative association with clinical stage and lymph node metastasis, which indicated that CD80 and CD86 detection may be useful for diagnosing and evaluating the prognosis of oral cancer. DCs, as the most potent type of antigen-presenting cell, express major histocompatibility complex (MHC) molecules and costimulatory molecules by capturing and processing antigens, prime initial $\mathrm{T}$ cells, initiate and regulate the immune response, and serve a key role in the induction of the antitumor response $(35,36)$. The abnormal number and function of DCs in neoplasms is regarded as an important factor in the immune evasion of tumors (37). Being members of the immunoglobulin superfamily, CD80 and CD86 are present on DCs in the form of oligomers (38). When the T-cell receptor-CD3 on T cells binds to the MHC class II antigen peptide of DCs, the T cells become stimulated and activated via the participation of costimulatory molecules in the immune response (39). In the absence of costimulatory molecules such as CD86 and CD80, DCs cannot deliver antigens effectively to T cells, leading to immune tolerance (35). The present study demonstrated that the expression levels of CD80 and CD86 were lower in the tissues of patients with advanced-stage cancer, which indicates that the lack of costimulatory molecules may provide a key mechanism for tumor immune evasion. Additionally, the abnormal differentiation of antigen-presenting cells and the accumulation of tolerant DCs may be the foundation of immune deficiency. According to Soloff and Barratt-Boyes (40), the influence of mucosal DCs on the formation of $\operatorname{IgA}$ in B cells is one of the causes of immune tolerance.

The present study identified a deficiency of DCs in tumors and the accumulation of IL-6 in saliva. A previous study supports the hypothesis that the abnormal quantity and function of DCs are closely associated with the excessive secretion of IL- 6 by tumor cells (41). When the body is in a pathological state, excessive IL-6 is secreted into the blood and body fluid, which inhibits the differentiation and maturation of DCs and maintains their immature state (42). When IL-6-specific monoclonal antibodies are neutralized, the function of DCs is restored (43).

The association between SIgA and DCs has been reported. The results of Bessa et al (44) indicated that lung mucosal DCs induce B cells to undergo a phenotypic conversion from $\operatorname{IgM}$ to IgA. DCs co-cultured with B and T cells promote the formation of SIgA (45). Mora et al (45) revealed that Peyer's patch DCs induce B cells to change the type of antibody they produce to IgA by secreting cytokines without relying on $\mathrm{T}$ cells. Therefore, it can be concluded that local DCs serve a unique role in the process of promoting the production of $\operatorname{Ig} \mathrm{A}$.

Based on the results of present and previous studies, tumor cells and the associated cytokines such as IL-6 are hypothesized to inhibit the function of DCs via autocrine or paracrine signaling when cancer occurs, thus it is difficult for DCs to promote the generation of SIgA by inducing the B cell phenotype conversion, and subsequently immune evasion arises. If the concentration of SIgA the oral cancer environment in increased via the administration of sustained-release tablets or injecting a submucosal SIgA vaccine, IL-6 production may be suppressed and DC immune deficiency may be partially reversed, thus preventing neoplasm development. Furthermore, neutralizing or reducing the concentration of IL-6 in the saliva may increase the level of SIgA and restore the function of DCs, therefore controlling the tumor. Although this hypothesis requires verification by additional studies, it provides a novel approach for clinical treatment.

However, certain issues remain. Although there are numerous advantages of biological indicators from saliva such as the stable composition, easy availability, noninvasive collection and repeated implementation (46), the detection index can be easily disturbed by factors such as inflammation, trauma, infection or mucosal lesion, which make clinical trials difficult to standardize and generate false positive results $(47,48)$.

In conclusion, the present study has shown that the concentrations of SIgA and IL-6 in the saliva may be used as auxiliary diagnostic indicators for oral cancer. The detection of CD80 and CD86 in tissues contributed to diagnosis and evaluation of the prognosis of tumors. Additional studies are required to test and verify that a SIgA vaccine or IL-6 inhibitor can be useful in reversing DC immune deficiency in oral cancer.

\section{Acknowledgements}

The present study was supported by the Key Technology Research Program of the Health Department of Hebei (grant no., 20100127) and the Science and Technology Planning Project of Hebei (grant no., 11276103D-71).

\section{References}

1. Warnakulasuriya S: Global epidemiology of oral and oropharyngeal cancer. Oral Oncol 45: 309-316, 2009.

2. Casto BC, Sharma S, Fisher JL, Knobloch TJ, Agrawal A and Weghorst CM: Oral cancer in Appalachia. J Health Care Poor Underserved 20: 274-285, 2009.

3. Ogbureke KU, Weinberger PM, Looney SW, Li L and Fisher LW: Expressions of matrix metalloproteinase-9 (MMP-9), dentin sialophosphoprotein (DSPP), and osteopontin (OPN) at histologically negative surgical margins may predict recurrence of oral squamous cell carcinoma. Oncotarget 3: 286-298, 2012.

4. Reizis B: Classical dendritic cells as a unique immune cell lineage. J Exp Med 209: 1053-1056, 2012.

5. Nudel I, Elnekave M, Furmanov K, Arizon M, Clausen BE, Wilensky A and Hovav AH: Dendritic cells in distinct oral mucosal tissues engage different mechanisms to prime CD8 + T cells. J Immunol 186: 891-900, 2011.

6. Corthesy B: Recombinant immunoglobulin A: Powerful tools for fundamental and applied research. Trends Biotechnol 20: 65-71, 2002.

7. Zhongying Niu: Basal and clinical of Oral Immunology. World Book Inc 98-136, 2001.

8. Kotani Y, Shinkai S, Okamatsu H, Toba M, Ogawa K, Yoshida H, Fukaya T, Fujiwara Y, Chaves PH, Kakumoto K and Kohda N: Oral intake of Lactobacillus pentosus strain b240 accelerates salivary immunoglobulin A secretion in the elderly: A randomized, placebo-controlled, double-blind trial. Immun Ageing 7: 11, 2010.

9. Sato A, Hashiguchi M, Toda E, Iwasaki A, Hachimura S and Kaminogawa S: CD11b+ Peyer's patch dendritic cells secrete IL-6 and induce IgA secretion from naive B cells. J Immunol 171: 3684-3690, 2003.

10. Bin G, Kewu H and Jiajia L: Preparation of single cell suspension from $\mathrm{r}$ - abbit muscle VX2 tumor with scissors versus homogenate method. Zhongguo zuzhi gongcheng yanjiu yu linchuang kangfu 11: 8315-8317, 2007.

11. De Schutter H, Landuyt W, Verbeken E, Goethals L, Hermans R and Nuyts S: The prognostic value of the hypoxia markers CA IX and GLUT 1 and the cytokines VEGF and IL 6 in head and neck squamous cell carcinoma treated by radiotherapy+/-chemotherapy. BMC Cancer 5: 42, 2005. 
12. Chen MF, Chen PT, Lu MS, Lin PY, Chen WC and Lee KD: IL-6 expression predicts treatment response and outcome in squamous cell carcinoma of the esophagus. Mol Cancer 12: 26, 2013.

13. Guo W, Dong Z, He M, Guo Y, Guo J, Chen Z, Yang Z and Kuang G: Aberrant methylation of thrombospondin-1 and its association with reduced expression in gastric cardia adenocarcinoma. J Biomed Biotechnol 2010: 721485, 2010.

14. Zhao Q, Zhang H, Li Y, Liu J, Hu X and Fan L: Anti-tumor effects of CIK combined with oxaliplatin in human oxaliplatin resistant gastric cancer cells in vivo and in vitro. J Exp Clin Cancer Res 29: 118, 2010.

15. Division of Oral and Maxillofacial Oncology; Chinese Society of Oral and Maxillofacial Surgery. The protocol of treatment guideline of oral and maxillofacial malignant neoplasms. China J Oral Practical Stomatology 8: 98-106, 2010.

16. Zhong LP, Zhang CP, Zhang ZY, et al: Survival analysis of 256 patients with oral cancer. Chin J Clin Oncol 42: 217-221, 2015.

17. Shin YS, Koh YW, Kim SH and Choi EC: Selective neck dissection for clinically node-positive oral cavity squamous cell carcinoma. Yonsei Med J 54: 139-144, 2013.

18. Reis PP, Waldron L, Perez-Ordonez B, Pintilie M, Galloni NN, Xuan Y, Cervigne NK, Warner GC, Makitie AA, Simpson C, et al: A gene signature in histologically normal surgical margins is predictive of oralcarcinoma recurrence. BMC Cancer 11: 437 , 2011.

19. Arellano-Garcia ME, Li R, Liu X, Xie Y, Yan X, Loo JA and Hu S: Identification of tetranectin as a potential biomarker for metastatic oral cancer. Int J Mol Sci 11: 3106-3121, 2010.

20. Shpitzer T, Bahar G, Feinmesser R and Nagler RM: A comprehensive salivary analysis for oral cancer diagnosis. J Cancer Res Clin Oncol 133: 613-617, 2007.

21. Brandtzaeg P: Role of secretory antibodies in the defence against infections. Int J Med Microbiol 293: 3-15, 2003.

22. Cai WQ and Wang BY: Digestive secretory IgA Practical immunocytochemis- and nucleic acid molecule hybridization techniques. Sichuan Science and Technology Publishing House, Chengdu, China, pp 190-230, 1994.

23. Cai WQ: Practical immunocytochemistry and nucleic acid molecule hybridization techniques. Sichuan Science and Technology Press, Chengdu, China, pp150-190, 1994.

24. Johansen FE, Braathen R and Brandtzaeg P: Role of $j$ chain in secretory immunoglobulin formation. Scand J Immunol 52: 240-248, 2000

25. Meng F, Yamagiwa Y, Ueno Y and Patel T: Over-expression of interleukin-6 enhances cell survival and transformed cell growth in human malignant cholangiocytes. J Hepatol 44: 1055-1065, 2006.

26. Wei LH, Kuo ML, Chen CA, Cheng WF, Cheng SP, Hsieh FJ and Hsieh CY: Interleukin-6 in cervical cancer: The relationship with vascular endothelial growth factor. Gynecol Oncol 82: 49-56, 2001.

27. Spiotto MT and Chung TD: STAT3 mediates IL-6-induced growth inhibition in the human prostate cancer cell line LNCap. Prostate 42: 88-89, 2000

28. Arellano-Garcia ME, Hu S, Wang J, Henson B, Zhou H, Chia D and Wong DT: Multiplexed immunobead-based assay for detection of oral cancer protein biomarkers in saliva. Oral Dis 14: 705-712, 2008

29. Sato J, Goto J, Murata T, Kitamori S, Yamazaki Y, Satoh A and Kitagawa Y: Changes in saliva interleukin-6 levels in patients with oral squamous cell carcinoma . Oral Surg Oral Med Oral Pathol Oral Radiol Endod 110: 330-336, 2010.

30. Ramsay AJ, Husband AJ, Ramshaw IA, Bao S, Matthaei KI, Koehler G and Kopf M: The role of interleukin-6 in mucosal IgA antibody responses in vivo. Science 264: 561-563, 1994.
31. Beagley KW, Bao S, Ramsay AJ, Eldridge JH and Husband AJ: IgA production by peritoneal cavity B cells is IL-6 independent: Implication for intestinal $\mathrm{IgA}$ responses. Eur J Immunol 25: 2123-2126, 1995

32. Strugnell RA and Wijburg OL: The role of secretory antibodies in infection immunity. Nat Rev Microbio1 8: 656-667, 2010

33. Mantis NJ and Forbes SJ: Secretory IgA: Arresting microbial pathogens at epithelial borders. Immunol Invest 39: 383-406, 2010

34. Corthësy B: Secretory immunoglobulin A: Well beyond immune exclusion at mucosal surfaces. Immunopharmacol ImmunotoxicoI 3: 174-179, 2009

35. Zou W: Immunosuppressive networks in the tumor environment and their therapeutic relevance. Nat Rev Cancer 5: 263-274, 2005.

36. Hao S, Bai O, Yuan J, Qureshi M and Xiang J: Dendritic cell-derived exosomes stimulate stronger CD8+CTL responses and antitumor immunity than tumor cell-derived exosomes. Cell Mol Immunol 3: 205-211, 2006.

37. Rutella S and Locatelli F: Targeting multiple-myeloma-induced immune dysfunction to improve immunotherapy outcomes. Clin Dev Immunol 2012: 196063, 2012.

38. Zhai SJ and $\mathrm{Su} \mathrm{CH}$ : Immunomodulatory function and clinical application of B7-CD28 family. Journal of Radiolimmuniology 21: 575 -578, 2008.

39. Li YK, Du JX, Lv ZH, et al: Research progress of CD80 and CD86 in tumor tissues and cells. Journal of Chinese Physician 17: 627-630, 2015.

40. Soloff AC and Barratt-Boyes SM: Enemy at the gates: Dendritic cells and immunity to mucosal pathogens. Cell Res 20: 872-885, 2010.

41. Ratta M, Fagnoni F, Curti A, Vescovini R, Sansoni P, Oliviero B, Fogli M, Ferri E, Della Cuna GR, Tura S, et al: Dendritic cells are functionally defective in multiple myeloma: The role of interleukin-6. Blood 100: 230-237, 2002.

42. Park SJ, Nakagawa T, Kitamura H, Atsumi T, Kamon H, Sawa S, Kamimura D, Ueda N, Iwakura Y, Ishihara K, et al: IL-6 regulates in vivo dendrtic cell differentiation through STAT3 activation. J Immunol 173: 3844-3854, 2004.

43. Wang S, Hong S, Yang J, Qian J, Zhang X, Shpall E, Kwak LW and Yi Q: Optimizing immunotherapy in multiple myeloma: Restoring the function of patients' monocyte-derived dendritic cells by inhibiting p38 or activating MEK/ERK MAPK and neutralizing interleukin-6 in progenitor cells. Blood 108: 4071-4077, 2006.

44. Bessa J, Jegerlehner A, Hinton HJ, Pumpens P, Saudan P, Schneider P and Bachmann MF: Alveolar macrophages and lung dendritic cells sense RNA and drive mucosal $\operatorname{IgA}$ responses. J Immunol 183: 3788-3799, 2009.

45. Mora JR, Iwata M, Eksteen B, Song SY, Junt T, Senman B, Otipoby KL, Yokota A, Takeuchi H, Ricciardi-Castagnoli P, et al: Generation of gut-homing IgA-secreting B cells by intestinal dendritic cell. Science 314: 1157-1160, 2006.

46. Cheng B, Rhodus NL, Williams B and Griffin RJ: Detection of apoptotic cells in whole saliva of patients with oral premalignant and malignant lesions: A preliminary study. Oral Surg Oral Med Oral pathol Oral Radiol Endod 97: 465-470, 2004.

47. Fraser CG: Inherent biological variation and reference values. Clin Chem Lab Med 42: 758-764, 2004.

48. Mankan AK, Lawless MW, Gray SG, Kelleher D and McManus R: NF-kappaB regulation: The nuclear response. J Cell Mol Med 13: 631-643, 2009 . 